\title{
Some Cross-Section Theorems on the Tangent Bundle over a Finslerian Manifold $\left(^{*}\right)(* *)$.
}

\author{
Tanjtro Okubo (Montreal, Canada) \\ C. S. HouH (Detroit, Michigan)
}

\begin{abstract}
Summary. - Let $T(M)$ be the tangent bundle over a Finslerian manifold $M$ of n-dimension en. dowed with the Cartan connection $\nabla$. One makes $T(M)$ into a $2 n$ dimensional affinely connected manifold by assigning a connection $\nabla^{\circ}$ to $T(M)$. The cross-section $\mathfrak{i}$ of a vector field $\nabla$ defined in $M I$ reveals in $T(M)$ an n-dimensional submanifold and its geometry is developed by means of the affine subspace theory and of the affine collineations in the base Finsler manifold.
\end{abstract}

\section{Introduction.}

K. YANo and OKUBO made an attempt to construct the geometry of Finslerian manifold by regarding it to be that of its tangent bundle itself [7]. In the theory of fibre bundles the notion of cross-section that provides the links between any object defined in base space and its image $\pi^{-1}$ in its bundle space plays the important role and this paper tries to discuss the geometrical properties of the cross-section of a vector field defined in the base FINSLER manifold.

$\S 1$ is devoted to the introduction of the tangent bundle $T(M)$ over a Finsler manifold $M$ with the CaRTaN connection $\nabla$. Herein endowing $T(M)$ with the vector fields $X^{\nabla}, X^{Z}$ and $X^{0}$ derived directly from the theory of connection and also by assigning the connection $\nabla^{c}, T(M)$ is made into an affinely connected manifold. $\$ 2$ deals with the infinitesimal affine collineations in FrNster manifolds, which together with $\S 1$ serves the main discussion presented in $\S 3$. Since the condition of complete integrability of affine collineations defined in Finslerian manifold is in our knowledge hard to find in any extant texts on the theory of Lre derivatives, it would not be less noteworthy to leave it in record (cf. (2.4)).

$\S 3$ discusses the geometry of the cross-seetion $\mathfrak{B}$ in $T(M)$ with respect to an arbitrary vector field $V(x)$ defined in $M$ along its differentiable curve $O$. $\mathfrak{B}$ reveals itself in $T(M)$ a submanifold with the dimension same as that of $M$ and along which we define the vertical, horizontal and complete vector fields denoted respectively by $X^{r}, X^{H}$ and $X^{c}$. Then we prove that $X^{P}$ lies in the plane normal to $\mathfrak{B}$ and $X^{H}$ is

(*) This work was supported by the National Research Council of Canada, 1970-1971, A.4037.

(**) Entrata in Redazione il giorno 8 maggto 1971 .

9 - Annali di Matematica 
tangential to $\mathfrak{B}$ if $\mathfrak{B}$ is a horizontal submanifold of $T(M)$, while $X^{c}$ is so if $\mathfrak{B}$ is horizontal and the Ire derivative of $X$ with respect to the vector field $V(x)$ vanishes. After establishing the subspace theory on $\mathfrak{B}$ we present the condition for the curvature vector field of $\mathfrak{B}$ to be tangential to $\mathfrak{B}$ (cf. Theorem 9 ).

\section{1. - Tangent bundle $T(M)$ over a Finslerian space.}

Let $M$ be an $n$-dimensional manifold of class $C^{\infty}$ and $T_{p}$ be the tangent plane at a point $P$ of $M$. Then $T(M)=\bigcup_{P \in A T} T_{p}(M)$ is by definition the tangent bundle over the base space $M$. A point $\tilde{P}$ of $T(M)$ is an ordered pair $\left(P, y_{P}\right)$ of a point $P$ of $M$ and a vector $y_{p} \in T_{p}(M) . \pi$ is the projection of $T(M)$ into $M$ defined by $\tilde{P} \rightarrow P$. The set $\pi^{-1}(P)$ is the fibre over $P$. On supposing that $M$ satisfies the second axiom of countability we introduce in $T(M)$ the local coordinate system $\left.x^{4}=\left(x^{i}, y^{i}\right){ }^{1}\right)$ in $\pi^{-1}\left\{U ;\left(x^{i}\right)\right\}$ where $U$ is a local coordinate neighbourhood of $P$ in $M$ and $y^{i}$ denote the components of vector $y$ at $p$ having the expression $y=y^{i} \partial_{i}$ with respect to the natural frame $\partial_{i}=\partial / \partial x^{i}$. Then, corresponding to the coordinate transformation $\left(x^{i}\right) \rightarrow\left(x^{i^{\prime}}\right)$ at $P \in\{U(x)\} \cap\left\{U^{\prime}\left(x^{\prime}\right)\right\},\left(x^{A}\right)$ obeys the law of transformation

$$
x^{A^{\prime}}=x^{A^{\prime}}\left(x^{B}\right)\left({ }^{1}\right)
$$

such that

$$
\begin{aligned}
& x^{i^{r}}=x^{i^{i}}\left(x^{i}\right), \text { det. }\left(\partial_{i} x^{i^{i}}\right) \neq 0, \\
& x^{\bar{i}^{i}}=y^{i^{i}}=\left(\partial_{i} x^{2^{i}}\right) y^{i}=\left(\partial_{i} x^{i^{i}}\right) x^{\bar{i}} .
\end{aligned}
$$

and its Jacobian matrix is given by

$$
\left(\partial_{\boldsymbol{B}} x^{A^{\prime}}\right)=\left[\begin{array}{cc}
\partial_{i} x^{h^{\prime}} & 0 \\
y^{a} \partial_{a} \partial_{i} x^{h^{\prime}} & \partial_{i} x^{h^{\prime}}
\end{array}\right] .
$$

We now take the direction $\dot{x}=d x / d t$ of any curve $C: x=x(t)$ of class $C^{r}, r \geqq 2$, as the fibre $y$ of $T(M)$ over $P$ and suppose that there is in $T(M)$ a differentiable function $L(x, \dot{x})$ which is positively homogeneous of degree one in the $\dot{x}^{\prime}$ s. On putting

$$
F(x, \dot{x})=L^{2}(x, \dot{x}) / 2
$$

(1) We adopt the following conventions for indices

$$
\begin{aligned}
& A, B, C, D=1,2, \ldots, n, \quad \overline{1}, \overline{2}, \ldots, \bar{n}, \\
& a, b, c, h, i, j, k=1,2, \ldots, n .
\end{aligned}
$$


and denoting $\partial / \partial \dot{x}^{i}$ by $\partial_{\vec{i}}$, we obtain $n^{2}$ symmetric functions $g_{i j}(x, \dot{x})$ which are homogeneous degree zero in the $\dot{x}^{\prime}$ s. If we assume that the quadratic form $g(A, A)$ defined for $\sum_{j=1}^{n} A^{j} A^{j}=1$ is positive definite, we call the base manifold $M$ a Finslerian space ([2], [3]). Then $T(M)$ is the tangent bundle over a Finsterian space $M$.

Since $T(M)$ is a $2 n$-dimensional manifold, we can introduce two complementary $n$-dimensional distributions $\tilde{V}$ and $\tilde{H}$ at $\pi^{-1} P$, called the vertical and horizontal subspaees. $\tilde{V}$ and $\tilde{H}$ are respectively spanned by $n$ independent base $\left(\partial_{i}\right)$ and

$$
\delta_{i}=\partial_{i}-\Gamma_{\imath}{ }^{k} \partial_{\bar{h}},
$$

where $\Gamma_{i}^{h}=\Gamma_{j i}{ }^{h} y^{j}$ and $\Gamma_{j i}{ }^{h}(x, \dot{x})$ are $u^{3}$ functions defined in $\pi^{-1}\left\{U ;\left(x^{i}\right)\right\}$ and undergo the law of tranformation subject to (1.1):

$$
\Gamma_{j^{\prime} i^{\prime}}^{h^{\prime}}=\left(\partial_{h} x^{h^{\prime}}\right)\left\{\partial_{j^{\prime}} \partial_{i^{\prime}} x^{h}+\left(\partial_{j^{\prime}}, x^{j}\right)\left(\partial_{i^{\prime}} x^{i}\right) \Gamma_{j i}^{h}\right\}
$$

We say that if there are given such $n^{3}$ functions in $T(M)$, the manifold is endowed with a non-linear connection $\nabla$ and $A$. KAWAGUCHI called $\delta_{i}$ the operator of basic connection. It was E. CARTAN who provided $M$ with $\nabla$ which is torsion-free and keeps the metric $g$ covariantly constant [7]

$$
\Gamma_{j i}^{h}(x, x)=\left\{\begin{array}{l}
h \\
j i
\end{array}\right\}-g^{h a}\left(\Gamma_{j}^{b} C_{b i a}+\Gamma_{i}^{b} C_{b j a}-\Gamma_{a}^{b} C_{b j i}\right),
$$

where $\left\{\begin{array}{l}h \\ j i\end{array}\right\}$ is the CHRISTOFFeL symbol formed with $g_{j i}$ and $C_{h j i}$ is defined by $\frac{1}{2} \partial_{\bar{h}} g_{j i}$ and satisfies

$$
C_{k j i} \dot{x}^{k}=C_{k j i} \dot{x}^{j}=C_{k j i} \dot{x}^{i}=0
$$

Hence we have for (1.5)

$$
\Gamma_{j i}^{h} \dot{x}^{j} \dot{x}^{i}=\Gamma_{i}^{h} \dot{x}^{i}=\left\{\begin{array}{l}
h \\
j i
\end{array}\right\} \dot{x}^{j} \dot{x}^{i}
$$

Also we have from (1.4)

$$
\left(\delta_{k} \delta_{j}-\delta_{j} \delta_{k}\right) f(x, \dot{x})=-K_{k j a}{ }^{h} y^{a} \partial_{\bar{h}} f(x, \dot{x})
$$

for any function $f(x, \dot{x})$ defined in $\pi^{-1} U$, where

$$
K_{k j i}^{n}=\delta_{k} \Gamma_{j i}^{n}-\delta_{j} \Gamma_{k i}^{h}+\Gamma_{k a}^{h} \Gamma_{j i}^{a}-\Gamma_{j a}^{h} \Gamma_{k i}^{a},
$$

and it is called the component of the curvature tesor of the CARTAN connection $\nabla$.

Let $X^{i}(x, \dot{x})$ be any $n$ functions that obey the law of transformation

$$
X^{h^{\prime}}\left(x^{\prime}, \dot{x}^{\prime}\right)=\left(\partial_{h} x^{h^{\prime}}\right) X^{h}(x, \dot{x}) .
$$


We define in $T(M)$ the vertical, horizontal and complete vector fields denoted respectively by $X^{p}, X^{R}$ and $X^{c}$ by [7]

$$
X^{v}=\left[\begin{array}{c}
0 \\
X^{h}
\end{array}\right], \quad X^{H}=\left[\begin{array}{c}
X^{h} \\
-\Gamma_{j i}^{h} y^{j} X^{i}
\end{array}\right], \quad X^{0}=\left[\begin{array}{c}
X^{h} \\
y^{j} \delta_{j} X^{h}
\end{array}\right],
$$

with respect to the natural frame $\partial_{A}=\left(\partial_{n}, \partial_{\bar{n}}\right)$ in $\pi^{-1}(U)$.

Likewise, we define for $n$ functions $\omega_{i}(x, x)$ satisfying

$$
\omega_{i^{\prime}}\left(x^{\prime}, x^{\prime}\right)=\left(\partial_{i^{\prime}} x^{3}\right) \omega_{i}(x, x)
$$

their vertical, horizontal and complete vector fields by

$$
\omega^{\gamma}=\left(\omega_{i}, 0\right), \quad \omega^{H}=\left(-\Gamma_{j i}^{h} y^{j} \omega_{h}, \omega_{i}\right), \quad \omega^{\sigma}=\left(y^{j} \delta_{j} \omega_{i}, \omega_{i}\right)
$$

Let $P$ and $Q$ be the two sets of $n^{r+s}$ and $n^{t+u}$ functions having in $\pi^{-1}(U)$ respectively the expression

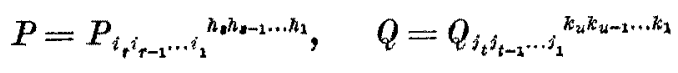

and obeying the law of transformation

$$
\begin{aligned}
& P_{r_{r}^{\prime} \ldots i_{1}^{\prime}} h_{s}^{\prime} \ldots h_{1}^{\prime}=\left(\partial_{i_{r}^{\prime}} x^{i_{r}}\right) \ldots\left(\partial_{i_{1}^{\prime}} x^{i_{1}}\right) \cdot\left(\partial_{h_{s}} x^{h_{s}^{\prime}}\right) \ldots\left(\partial_{h_{1}} x^{h_{1}^{\prime}}\right) P_{i_{r} \ldots i_{1}}^{n_{g} \ldots h_{1}},
\end{aligned}
$$

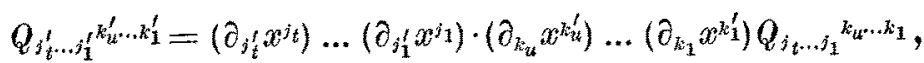

subject to (1.1). Denoting by $\mathrm{P} \times Q$ the formal functional product of $P$ and $Q$, we define the vertical, horizontal and complete tensor fields respectively by

$$
\left\{\begin{array}{l}
(P \times Q)^{\nabla}=P^{\nabla} \otimes Q^{\nabla}, \\
(P \times Q)^{R}=P^{\boldsymbol{B}} \otimes Q^{\nabla}+P^{\nabla} \otimes Q^{B} \\
(P \times Q)^{\sigma}=P^{\sigma} \otimes Q^{\nabla}+P^{\nabla} \otimes Q^{c}
\end{array}\right.
$$

By the use of the CARTAx connection $\nabla$ we introduce in $T(M)$ the affine connection $\nabla^{o}$ so that $T(M)$ is now made into an affine manifold. $\nabla^{c}$ is supposed to have in $\pi^{-1} U$ the components of the form [6]

$$
\begin{aligned}
& \tilde{\Gamma}_{j i}^{h}=\Gamma_{j i}^{n}, \tilde{\Gamma}_{\bar{j} i}^{n}=\tilde{\Gamma}_{j \bar{i}}^{h}=\tilde{\Gamma}_{\overline{j i}}^{n}=0, \\
& \tilde{\Gamma_{j i}^{n}}=y^{a} \delta_{a} \Gamma_{j i}^{h}, \tilde{\Gamma_{j i}^{n}}=\tilde{\Gamma_{j \bar{i}}^{n}}=\Gamma_{j i}^{n}, \tilde{\Gamma}_{j \bar{i}}^{\bar{n}}=0 .
\end{aligned}
$$


Let $X(x, \dot{x})$ and $Y(x, \dot{x})$ be any vector fields in $T(M)$.

$$
\begin{aligned}
\nabla_{X^{c}}^{o} Y^{c}=\left[\begin{array}{c}
X^{j} \nabla_{j} Y^{h} \\
\dot{x}^{a} \delta_{a}\left(X^{j} \nabla_{j} y^{h}\right)
\end{array}\right]- \\
-\left[\begin{array}{c}
0 \\
K_{k^{j} i}{ }^{a} X^{k} \dot{x}^{j} \dot{x}^{i} \partial_{\bar{a}} Y^{h}
\end{array}\right]-\left[\begin{array}{c}
0 \\
\left(\dot{x}^{a} \nabla_{a} X^{i}\right)\left\{\dot{x}^{b} \partial_{b} \partial_{\bar{i}} Y^{h}+\dot{x}^{b} \partial_{\dot{i}}\left(\Gamma_{b}^{a} \partial_{\bar{a}} Y^{h}\right)\right\}
\end{array}\right]
\end{aligned}
$$

where $\nabla_{j} Y^{h}$ is defined by

$$
\nabla_{j} Y^{h}=\delta_{j} \nabla^{h}+\Gamma_{j i}^{h} \nabla^{i}
$$

and as we have by (1.8),

$$
\left(\nabla_{X} Y\right)^{a}=\left[\begin{array}{c}
X^{j} \nabla_{j} Y^{h} \\
\dot{x}^{a} \delta_{a}\left(X^{j} \nabla_{j} Y^{h}\right)
\end{array}\right],
$$

we can state

THEOREM 1. $-\nabla_{x^{c}}^{c} Y^{c}$ coincides with $\left(\nabla_{x} Y\right)^{c}$ if $Y$ does not depend upon the $\dot{x}^{\prime}$ s. The curvature tensor $K^{c}$ of $\nabla^{0}$ is by definition given by

$$
K^{o}(\tilde{X}, \tilde{Y}) \tilde{Z}=\nabla_{\tilde{X}}^{o} \nabla_{\tilde{Y}}^{c} \tilde{Z}-\nabla_{\tilde{Y}}^{c} \nabla_{\tilde{X}}^{o} \tilde{Z}-\nabla_{[\tilde{X}, \tilde{y}]}^{o} \tilde{Z}
$$

where $\tilde{X}, \tilde{Y}$ and $\tilde{Z}$ are any vector fields in $T(M)$, and for the variable range of indices $K^{c}$ is found to have in $\pi^{-1}(U)$ the components of the form given by

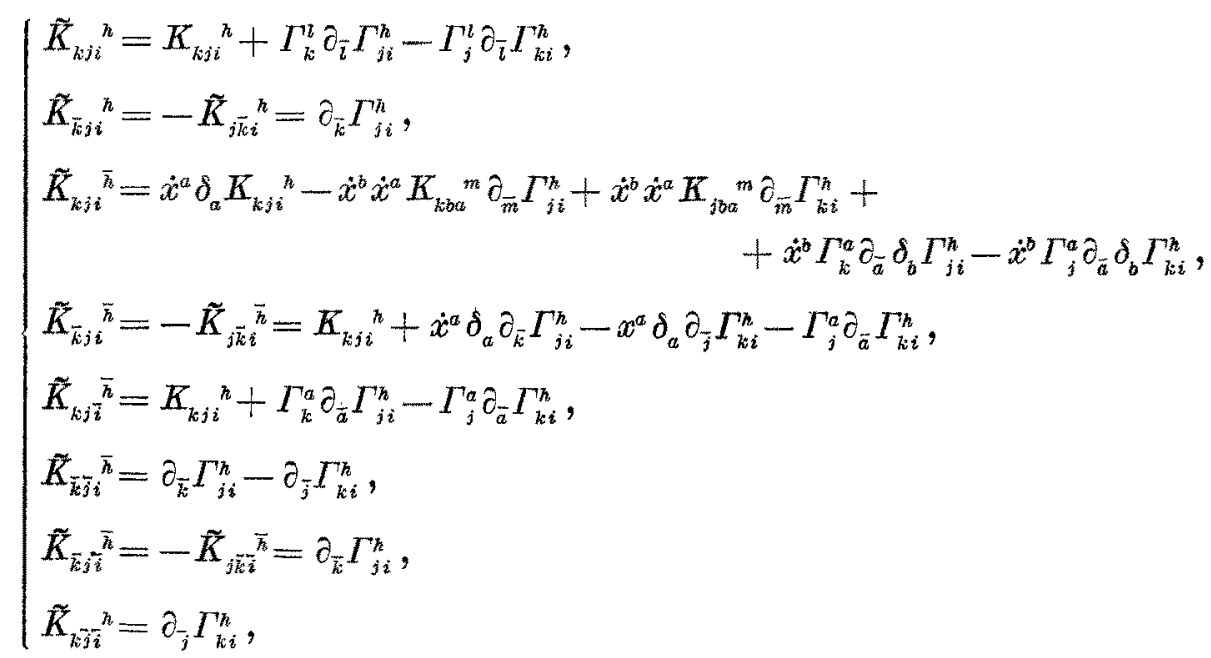

all other being zero. 
On computing the BIANCHI identities

$$
\begin{aligned}
& K^{c}(\tilde{X}, \tilde{Y}) \tilde{Z}+K^{c}(\tilde{Y}, \tilde{Z}) \tilde{X}+K^{c}(\tilde{Z}, \tilde{X}) \tilde{Y}=0 \\
& \nabla_{\tilde{X}}^{c} K^{c}(\tilde{Y}, \tilde{Z}) \tilde{W}+\nabla_{\tilde{Y}}^{c} K^{c}(\tilde{Z}, \tilde{X}) \tilde{W}+\nabla_{\tilde{Z}}^{c} K^{c}(\tilde{X}, \tilde{Y}) \tilde{W}=0
\end{aligned}
$$

we have

THEOREM 2. - The curvature tensor $K$ of the Cartan connection satisfies

$$
\begin{aligned}
& K_{k j i^{h}}+K_{j: k_{i}}{ }^{n}+K_{i k j^{n}}=0 \\
& \nabla_{l} K_{k j j^{h}}{ }^{h}+\nabla_{k} K_{j l i}{ }^{h}+\nabla_{i} K_{l k i}{ }^{h}=-K_{l k b}{ }^{a} \dot{x}^{b} \partial_{\bar{a}} \Gamma_{j i}^{h}-K_{k j b b}{ }^{a} \dot{x}^{b} \partial_{\bar{u}} \Gamma_{l i}^{h}-K_{j l b}{ }^{a} \dot{x}^{b} \partial_{\bar{a}} \Gamma_{k i}^{h} .
\end{aligned}
$$

The formulas (1.14) and (1.15) are called the BravcHI identities of the first and second kinds respectively [2].

\section{2. - Infinitesimal affine collineations.}

Since $T(T(M))$ is spanned by $\tilde{V}$ and $\tilde{H}$ determined respectively by $\left(\partial_{i}\right)$ and $\left(\delta_{i}\right)$, the cotangent plane ' $T(T(M))$ is spanned by the two planes dual to $\widetilde{V}$ and $\tilde{H}$ and their base are respectively given by $\left(\delta x^{i}\right)$ and $\left(d x^{i}\right)$, where

$$
\delta x^{i}=d x^{i}+\Gamma_{j}^{i} x^{j} .
$$

Let $C: x=x(t)$, be a curve of class $C^{r}, r \geq 2$, and $\dot{x}$ be its direction. If in $T(M)$ the 1-form (2.1) vanishes along $C$ so that we have

$$
\frac{\mathrm{d}^{\mathrm{a}} x^{x^{2}}}{\mathrm{~d} t^{2}}+\Gamma_{j i}^{h} \frac{\mathrm{d} x^{i}}{\mathrm{~d} t} \frac{\mathrm{d} x^{i}}{\mathrm{~d} t}=0
$$

we call $O$ the geodesic of $M$. Let $V(x)$ be any vector field defined along $C$ in $M$ and consider the infinitesimal transformation

$$
\begin{aligned}
& \bar{x}^{i}=x^{i}+V^{i}(x) \delta u \\
& \bar{x}^{i}=\dot{x}^{i}+\dot{x}^{a} \partial_{a} V^{i} \delta u .
\end{aligned}
$$

Then we say that if (2.2) sends the geodesic $O$ to a geodesic, the vector field $V(x)$ generates an affine collineation [7], and its necessary and sufficient condition is given by the vanishing of the LII derivative $\mathfrak{L}_{\nabla} \Gamma_{j i}^{h}$ that is,

$$
\mathfrak{L}_{V} \Gamma_{j i}^{h}=\nabla_{j} \nabla_{i} V^{h}+K_{k j i}{ }^{h} V^{k}+\left(\dot{x}^{b} \nabla_{b} V^{a}\right) \partial_{\bar{a}} \Gamma_{j i}^{h}=0
$$


and the condition of integrability of the equations is found by a straightforward computation to be

$$
\nabla_{k} \mathfrak{R}_{V} \Gamma_{j i}^{h}-\nabla_{j} \mathfrak{R}_{V} \Gamma_{k i}^{h}=\mathfrak{L}_{V} K_{k j i}{ }^{h}+\left(\dot{x}^{b} \mathfrak{Q}_{V} \Gamma_{k b}^{a}\right) \partial_{\bar{a}} \Gamma_{j i}^{h}-\left(\dot{x}^{b} \mathfrak{Q}_{V} \Gamma_{j b}^{a}\right) \partial_{\bar{a}} \Gamma_{k i}^{h}=0
$$

Hence we have by taking account of (2.3)

THeOReM 3. - The condition of integrability of $\mathfrak{L}_{V} \Gamma_{i i}^{h}=0$ is given by the vanishing of $\mathfrak{Q}_{F} K_{k j i}{ }^{k}$.

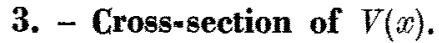

Let $V(x)$ be a vector field of $M$ defined along a curve $C: x=x(t)$, of class $C^{r}$, $r \geqq 2$, that lies in $\left\{U ;\left(x^{i}\right)\right\}$. The coordinates $x^{4}$ of $\pi^{-1}(P \in C)$ in $\pi^{-1}(U)$ corresponding to the vector $V(x)$ issuing from $P$ is then given by

$$
x^{A}=\left(x^{i}, V^{i}(x)\right)
$$

which we call the cross-section of $V(x)$ [1], [4] and it reveals itself in $T(M)$ an $n$-dimensional submanifold. Hereafter we express it by $\mathfrak{B}$. Along $\mathfrak{B}$ we have from (3.1)

$$
\dot{x}^{A}=\left(\dot{x}^{i}, \dot{x}^{a} \partial_{a} V^{i}\right)
$$

and with those $\dot{x}^{i}$ involved in $x^{A}$ the $n^{3}$ functions $\Gamma_{j i}^{h}(x, \dot{x})$ of $T(M)$ are well defined throughout $\tilde{V}$. Subject to these $\Gamma_{j i}^{h}(x, \dot{x})$ if the vector field $V(x)$ satisfies

$$
\nabla_{j} V^{h}=\partial_{j} \nabla^{h}+\Gamma_{j i}^{n}(x, \dot{x}) V^{i}=0
$$

we say that $\mathfrak{B}$ is the horizontal submanifold of $T(M)$.

The base $B$ of the tangent plane of $\mathfrak{B}$ has in $\pi^{-1}(U)$ the expression

$$
B_{i}{ }^{A}=\partial_{i} x^{A}=\left[\begin{array}{c}
\delta_{i}{ }^{n} \\
\partial_{i} V^{h}
\end{array}\right],
$$

and serves to map any vector field defined in $\mathfrak{B}$ into a vector in $T(M)$ tangent to $\mathfrak{B}$. In order to obtain the WHITNEY sum of frames at each point of $\mathfrak{B}$, we choose $n$ affine normals $C_{\bar{i}}^{A}$ defined by

$$
C_{\bar{i}}^{A}=\partial x^{A} / \partial V^{i}=\left[\begin{array}{l}
0 \\
\delta_{i}^{h}
\end{array}\right]
$$


Then the coframe $\left(B_{B}^{h},{C^{\bar{h}}}_{B}\right)$ dual to the frame $\left(B_{i}^{A}, C_{\bar{i}}^{A}\right)$ has the expression

$$
B^{h}{ }_{B}=\left(\delta_{i}^{h}, 0\right), \quad{C^{\bar{h}}}_{B}=\left(-\partial_{i} V^{h}, \delta_{i}^{h}\right) .
$$

Let $X^{n}(x, \dot{x})$ be any $n$ functions in $T(M)$ defined along $\mathfrak{P}$ satisfying the law of transformation given in (1.7), and by the use of which we construct $X^{r}$. If we decompose $X^{\nabla}$ into the directions tangent and normal to $\mathfrak{B}$, we have

$$
B^{h}{ }_{4}\left(X^{\nabla}\right)^{A}=0, \quad C^{\bar{h}}\left(X^{\nabla}\right)^{A}=X^{h}
$$

in virtue of (3.5). Hence we have

$$
X^{V}=C_{\vec{i}}^{A} X^{i},
$$

and thus

THEOREM 4. - Any vertical veetor field defined along $\mathfrak{B}$ is ontained in the normale plane of $\mathfrak{B}$.

Along $\mathfrak{2}$ we define $X^{H}$ and $X^{o}$ by using the above stated $n$ functions $X^{n}(x, \dot{x})$ and $n^{3}$ functions $\Gamma_{j i}^{h}(x, \dot{x})$ by

$$
X^{H}=\left[\begin{array}{c}
X^{h} \\
-\Gamma_{j i}^{h}(x, \dot{x}) \nabla^{j} X^{i}
\end{array}\right], \quad X^{o}=\left[\begin{array}{c}
X^{h} \\
V^{j} \delta_{j} X^{h}
\end{array}\right],
$$

respectively. Then we have

$$
B^{h}{ }_{4}\left(X^{B}\right)^{A}=X^{h}, \quad C^{\bar{h}}\left(X^{H}\right)^{A}=-\left(\nabla_{i} V^{h}\right) X^{i},
$$

from which we get

$$
\left(X^{B}\right)^{A}=B_{i}^{A} X^{i}-O_{i}^{A}\left(\nabla_{j} \nabla^{i}\right) X^{j}
$$

and hence we have by taking account of (3.2)

THEOREM 5. - Any horizontal veetor field defined along $\mathfrak{B}$ is tangential to $\mathfrak{B}$ if $\mathfrak{B}$ is an horizontal submanifold of $T(M)$.

As for $X^{\circ}$ we have

$$
B^{h}{ }_{A}\left(X^{\sigma}\right)^{A}=X^{h}, \quad C^{\vec{h}}{ }_{A}\left(X^{\sigma}\right)^{A}=\mathfrak{I}_{\nabla} X^{h}-\left(\dot{x}^{b} \nabla_{b} V^{a}\right) \partial_{\bar{a}} X^{h}
$$

from which we get

$$
\left(X^{c}\right)^{A}=B_{i}^{A} X^{i}+O_{\vec{i}}^{A}\left\{\mathfrak{S}_{V} X^{i}-\left(\dot{x}^{b} \nabla_{b} V^{a}\right) \partial_{\bar{a}} X^{i}\right\} .
$$

Thus we have

THEOREM 6. - Any complete vector field $X^{o}$ defined along $\mathfrak{B}$ is tangential to $\mathfrak{B}$ if the Lre derivative $\mathfrak{R}_{V} X$ vanishes and $\mathfrak{B}$ is a horizontal submanifold of $T(M)$. 
We have introduced in $T(M)$ the affine connection $\nabla^{0}$ whose components in $\pi^{-1}(U)$ were given in (1.9). Then we have for $\mathfrak{B}$ the van der WAERDEN-BORTOLOTTIE equations

$$
\nabla_{B \bar{X}}^{o} B \bar{Y}=B^{\prime} \nabla_{\bar{X}} \bar{Y}+H^{i}(\bar{X}, \vec{Y}) C_{\bar{i}}
$$

where $\bar{X}$ and $\bar{Y}$ are arbitrary vector fields of $\mathfrak{B}$ and ${ }^{\prime} \nabla$ is the connection of $\mathfrak{B}$, induced from $\nabla^{c}$ which is symmetric and is called the induced connection of $\mathfrak{B}$. $H^{i}$ are $n$ symmetric tensor fields of type $(0.2)$ and are called the second fundamental tensor fields of $\mathfrak{B}$. We say that $\mathfrak{B}$ is a totally geodesic submanifold of $T(M)$ if $H^{i}$ vanish identically. In terms of the local coordinates ' $\nabla$ has the components

$$
{ }^{\prime} \Gamma_{i j}^{h}(x, \infty)=\left(\partial_{j} B_{i}{ }^{4}+\left(\Gamma^{A}{ }^{A}\right)^{o} B_{j}{ }^{c} B_{i}{ }^{B}\right) B^{n}{ }_{A}
$$

where $\left(\Gamma_{o_{B}}^{A}\right)^{\sigma}$ denote the components of $\nabla^{o}$ with respect to $\left(x^{A}\right)$ of $\pi^{-1}(U)$. Then taking account of (1.9), (3.3) and (3.5) we find

$$
T_{j i}^{h}=\Gamma_{j i}^{h}
$$

and $(3.10)$ is reducible to

$$
{ }^{\prime} \nabla_{j} B_{i}^{A}=\left\{\mathfrak{Q}_{V} \Gamma_{j i}^{h}-\left(\dot{x}^{b} \nabla_{b} \nabla^{a}\right) \partial_{\bar{a}} \Gamma_{j i}^{h}\right\} C_{\bar{h}}^{A}
$$

Hence we have by taking account of (2.3) and (3.2)

THEOREM 7. - The oross-seotion $\mathfrak{B}$ of a vector field $V(x)$ is a totally geodesic submanifold, if $V(x)$ generates an affine collineation in $M$ and $\mathfrak{B}$ is a horizontal submanifolds in $T(M)$.

The local expression of the WEINGARTEN equations of $\mathfrak{B}$ is given by

$$
{ }^{\prime} \nabla_{j} C_{\bar{i}}^{A}=\partial_{j} C_{\bar{i}}^{A}+\left(\Gamma_{o B}^{A}\right)^{o} B_{j}^{o} C_{\bar{i}}^{B}-{ }^{\prime} T_{j \bar{i}}^{h} C_{\bar{h}}^{A}
$$

and by taking account of (1.9), (3.3) and (3.11) we find

$$
{ }^{\prime} \nabla_{j} C_{\tilde{i}}^{A}=0
$$

Thus

THEOREM 8. - The affine normal vector fields $C_{\overline{\boldsymbol{i}}}$ are transported parallel along $\mathfrak{B}$.

Since we have (3.10) the structure equations of $\mathfrak{B}$ will only be given by

$$
\nabla_{B \bar{X}}^{o} \nabla_{B \bar{Y}}^{o} B \bar{Z}-\nabla_{B \bar{Y}}^{c} \nabla_{B \bar{X}}^{o} B \bar{Z}-\nabla_{[\bar{B} \cdot, B \bar{Y}]}^{o} B \bar{Z}=K^{c}(B \bar{X}, B \bar{Y}) B \bar{Z}
$$


and it has the local expression

$$
{ }^{\prime} \nabla_{k}{ }^{\prime} \nabla_{j} B_{i}{ }^{A}-{ }^{\prime} \nabla_{j} \nabla_{k} B_{i}{ }^{A}=(K)_{D C B}^{c}{ }^{A} B_{k}{ }^{D} B_{j}{ }^{\alpha} B_{i}{ }^{B}-K_{k j i}{ }^{h} B_{h}{ }^{A}
$$

in virtue of (3.10) and (3.11). If we take account of the components $(K)_{D C B}^{c}{ }^{A}$ of $K^{o}$ given in (1.13) and also of (3.3), the right hand side of (3.14) is found to have the form

$$
\begin{aligned}
& {\left[\mathfrak{R}_{V} K_{k j i}{ }^{h}+\left(\dot{x}^{b} \mathfrak{I}_{V} \Gamma_{k b}^{a}\right) \partial_{\bar{a}} \Gamma_{j i}^{h}-\left(\dot{x}^{b} \mathfrak{\Omega}_{V} \Gamma_{j b}^{a}\right) \partial_{\bar{a}} \Gamma_{k i}^{h}-\right.} \\
& -\dot{x}^{d}\left\{\partial_{\bar{b}} K_{k j i}{ }^{h}+\left(\partial_{\bar{b}} \Gamma_{k c}^{a}\right) \dot{x}^{c} \partial_{\bar{a}} \Gamma_{j i}^{h}-\left(\partial_{\bar{b}} \Gamma_{j c}^{a}\right) \dot{x}^{c} \partial_{\bar{a}} \Gamma_{k i}^{h}\right\} \nabla_{\bar{d}} V^{b}- \\
& \left.\quad-\dot{x}^{a}\left\{\left(\partial_{\bar{b}} \Gamma_{j i}^{h}\right) \nabla_{k} \nabla_{a} V^{b}-\left(\partial_{b} \Gamma_{k i}^{h}\right) \nabla_{j} \nabla_{a} V^{b}\right\}\right] C_{\bar{h}}{ }^{A}
\end{aligned}
$$

Hence we have by taking account of (2.3), (3.2), (3.14), (3.15) and also of Theorem 4:

THEOREM 9. - Let $\bar{X}, \bar{Y}$ and $\bar{Z}$ be any vector fields in $\mathfrak{B}$, then the curvature vcctor $K^{\circ}(B \bar{X}, B \bar{Y}) B \bar{Z}$ is tangential to $\mathfrak{B}$, if $\mathfrak{B}$ is a horizontal submanifold in $T(M)$ and $V(x)$ defines an affine collineation in $M$.

\section{REFERENCES}

[1] T. Oкuво, Cross-section theorems on the tangent bundle over a generalized space of paths, to appear in Revue Roumaine Math. pures et applic.

[2] H. RuND, The differential geometry of Finsler spaces, Springer-Verlag, Berlin, 1959.

[3] K. Yano, The theory of Lie derivatives and its application, North Holland Publ., Amsterdam, 1957.

[4] K. YANo, Tensor fields and connections on cross-sections in the tangent bundle of a differentiable manifold, Proc. Royal Soc. of Edinburgh, 67 (1967), pp. 277-288.

[5] K. YANO - S. KoBAYASHT, Prolongations of tensor fields and connections to tangent bundles, I, Journ. Math. Soc. Japan, 18 (1966), pp. 194-210; II, ibid., 18 (1966), pp. 236-246.

[6] K. Y YNO - T. OKuBo, On the tangent bundles of generalized spaces of paths, to appear in Rendic. di Mat., Roma.

[7] K. YANo - T. OKuBo, On the tangent bundles of Finsler and Riemann manifolds with Sasaki metrics, Annali di Mat. Pura ed Appl., 77 (1970), pp. 137-162. 